\title{
Eficacia de amodiaquina y sulfadoxina/pirimetamina en el tratamiento de malaria no complicada por Plasmodium falciparum en Nariño, Colombia, 1999-2002
}

\begin{abstract}
Iveth J. González ${ }^{1}$, Julio C. Padilla ${ }^{2}$, Luis E. Giraldo † ${ }^{1}$, Nancy G. Saravia ${ }^{1}$
${ }^{1}$ Centro Internacional de Entrenamiento e Investigaciones Médicas, CIDEIM, Cali, Colombia.

2 Programa Nacional de Control de Enfermedades Transmitidas por Vectores, Ministerio de Salud, Bogotá, D.C., Colombia.

La resistencia a los antimaláricos es una de las causas del aumento de casos de malaria en el mundo. Desde el año 2000, el tratamiento de malaria no complicada por Plasmodium falciparum en Colombia ha sido la combinación de amodiaquina (AQ) y sulfadoxina/pirimetamina (SP). La eficacia de estos dos medicamentos se evaluó después de la implementación del nuevo esquema. El estudio se realizó en los municipios de El Charco y Tumaco (Nariño) en la Costa Pacífica. Se utilizó el protocolo estándar de la OPS para la evaluación de la eficacia de antimaláricos en áreas de baja a moderada transmisión. Los pacientes incluidos fueron asignados al azar a los dos medicamentos de estudio y seguidos hasta el día 14 en El Charco y hasta el día 28 en Tumaco. Ninguno de los 48 pacientes en El Charco presentó falla terapéutica a los medicamentos en estudio. En Tumaco, por el contrario, 12 de 24 pacientes (IC95\%: 30,6 a 69,4) presentaron falla a la AQ y 4 de 26 (IC95\%:5,1-33,1) presentaron falla a SP. Los altos niveles de falla a AQ en Tumaco fueron inesperados por su reciente introducción oficial al tratamiento de malaria, mientras que los niveles de falla a SP aumentaron respecto a lo encontrado en estudios anteriores. Estos hallazgos sugieren que el uso de $\mathrm{AQ}$ a las dosis actuales en combinación con SP tendrá un tiempo de vida útil más corto que el esperado. El uso combinado de antimaláricos como estrategia para retardar la aparición de resistencia será efectivo en la medida en que las monoterapias sean eficaces.
\end{abstract}

Palabras clave: Plasmodium falciparum, antimaláricos, amodiaquina, sulfadoxina-pirimetamina, resultado del tratamiento.

Efficacy of amodiaquine and sulfadoxine/pyrimethamine in treating uncomplicated cases of Plasmodium falciparum malaria (Nariño, Colombia, 1999-2002)

The resurgence and spread of antimalarial drug resistance is one of the causes of the worldwide increase of malaria. In Colombia, uncomplicated Plasmodium falciparum malaria has been treated with a combination of amodiaquine(AQ) and sulfadoxine/pyrimethamine(SP) since 2000 . The efficacy of these two antimalarials was evaluated after the implementation of the new malaria treatment scheme. In the municipalities of El Charco and Tumaco (Nariño) on the Pacific Coast region, the standard PAHO protocol was used to evaluate antimalarial efficacy in areas of low to moderate malaria transmission. Patients were randomly allocated to treatment regime in two cities of Nariño, El Charco $(n=48)$ and Tumaco $(n=50)$. After 14 days none of El Charco patients presented therapeutic failure to either antimalarial. However, in Tumaco after 28 days, 12 of 24 (95\% Cl:30.6-69.4) patients presented AQ treatment failure while 4 of 26 (95\%Cl:5.1-33.1) patients had SP treatment failure. The high level of $A Q$ treatment failure in Tumaco was unexpected because it had been introduced only recently as an antimalarial treatment in Colombia. The results suggest that the use of the current dose of $A Q$ in combination with SP will be therapeutically useful for less time than expected. Use of combined therapies is a key strategy to delay antimalarial resistance. Unfortunately, its success depends on the efficacy of antimalarial drugs individually.

Key words: Plasmodium falciparum, antimalarials, amodiaquine, sulfadoxine-pyrimethamine, treatment failure. 
La resistencia de Plasmodium falciparum a los medicamentos de uso más común, tales como cloroquina (CQ) y sulfadoxina/pirimetamina (SP), es un fenómeno que afecta a casi todos los países con transmisión de malaria y ha ido en aumento progresivo durante los últimos 20 años. La resistencia a los antimaláricos ha sido considerada como una de las principales causas del aumento de casos y muertes por malaria alrededor del mundo y del aumento en la frecuencia y gravedad de las epidemias (1). En América son muy pocos los países que en la actualidad siguen usando $\mathrm{CQ}$ como medicamento de primera línea en el tratamiento de malaria por $P$. falciparum y sólo en algunos de ellos se sigue usando SP, pero en combinación con otros antimaláricos (2).

En Colombia, la mayor parte de los casos de malaria son producidos por Plasmodium vivax; sin embargo, en la Costa Pacífica, alrededor del $80 \%$ de los casos son producidos por P. falciparum (3). Nariño, ubicado al sur de la Costa Pacífica, es uno de los departamentos que más casos de malaria por $P$. falciparum informa anualmente y en donde se presenta la mayoría de casos de muerte por esta enfermedad. En 2001 se presentaron 25 muertes por malaria en este departamento y 30.996 casos de malaria, de los cuales el $88,3 \%$ fue causado por $P$. falciparum (4). Uno de los factores que podría estar contribuyendo al empeoramiento de la situación de malaria en la Costa Pacífica es la disminución en la eficacia de los antimaláricos.

Estudios realizados al norte de la Costa Pacífica y en el departamento de Antioquia, alrededor de 1998, demostraron la existencia de altos niveles de fallas terapéuticas a CQ: 44\% en Quibdó, Chocó (5); $67 \%$ en Zaragoza, Antioquia (6); 78\% en Cali, Valle, (pacientes de la Costa Pacífica) (7), y $97 \%$ en Turbo, Antioquia (8). Teniendo en

† Este artículo ha sido dedicado a la memoria de Luis Ernesto Giraldo q.e.p.d.

Correspondencia:

Iveth J. González, Centro Internacional de Entrenamiento e Investigaciones Médicas (CIDEIM). Avenida 1N \# 3-03, Cali, Colombia. Teléfono: (572) 668 2164. Fax: (572) 6672989. Correo electrónico: ivethgon@cideim.org.co.

Recibido: 28/10/02; aceptado: 14/02/03 cuenta estos datos, el Ministerio de Salud, con la asesoría de un grupo de expertos en malaria, recomendó reemplazar la $\mathrm{CQ}$ por amodiaquina (AQ) en combinación con SP para el tratamiento de primera línea de la malaria no complicada por P. falciparum en Colombia a partir del año 2000 (3). Sin embargo, en ese entonces se conocía poco respecto a la eficacia de $A Q$ en Colombia. En la actualidad, después de la implementación del nuevo esquema de tratamiento, se desconoce cuál es la eficacia de $A Q$ y $S P$ en las áreas endémicas de Colombia en donde predomina $P$. falciparum.

La SP ha sido utilizada a nivel mundial como la segunda opción de tratamiento de malaria después de $C Q$ y en algunos países de Africa se viene utilizando como tratamiento de primera línea gracias a su bajo costo y alta eficacia (1). La larga vida media de SP ha favorecido la presión de selección y el rápido surgimiento de cepas de $P$. falciparum resistentes, por lo cual se ha recomendado su uso en combinación con otros antimaláricos como estrategia de retardo de la aparición de resistencia (9). Aunque la SP se ha usado en Colombia desde 1981, los estudios realizados en 1998 demostraron bajos niveles de falla a este medicamento: 6\% en Quibdó, Chocó, (5), $9 \%$ en Zaragoza, Antioquia, (6) y $12 \%$ en Turbo, Antioquia (8). Los bajos niveles de fallas terapéuticas a SP en Colombia han permitido seguir recomendando su uso en combinación con $\mathrm{AQ}$.

La $A Q$ es una 4-aminoquinolina análoga a la $C Q$ que ha demostrado ser eficaz en el tratamiento de malaria en áreas con alta resistencia a $\mathrm{CQ}$ (10). Los estudios realizados en Colombia antes de la introducción de este medicamento al esquema oficial de tratamiento de malaria, mostraron bajos niveles de resistencia in vitro y de fallas terapéuticas in vivo. Un estudio realizado en 1985, en el cual se evaluó la sensibilidad in vitro de aislamientos de campo de $P$. falciparuma $A Q$, demostró que $1 / 30(3,3 \%)$ aislamientos fue resistente al medicamento (11). Por otro lado, dos estudios realizados en Antioquia en 1998, en los cuales se evaluó la eficacia de $A Q$ en el tratamiento de malaria demostraron que sólo el $3 \%$ (en Zaragoza) y el 7\% (en Turbo) de los 
pacientes tratados presentaron falla terapéutica al medicamento $(6,8)$. A pesar de la eficacia demostrada de $A Q$, su analogía con $C Q$ y la posibilidad de resistencia cruzada con este medicamento permiten especular que el tiempo de vida útil de $A Q$ en el tratamiento de malaria en áreas con alta resistencia a $C Q$ es corto $(12,13)$.

El objetivo del presente estudio fue determinar la frecuencia de fallas terapéuticas a $A Q$ y a SP en pacientes con malaria no complicada por $P$. falciparum en dos municipio de Nariño, en la Costa Pacífica de Colombia. Al mismo tiempo, este estudio ofreció la oportunidad de entrenar personal de los programas locales de control de malaria en la metodología de evaluación in vivo de eficacia de antimaláricos similar a la utilizada actualmente en los estudios de la Red Nacional de Farmacovigilancia en Malaria (14).

\section{Materiales y métodos}

\section{Tipo de estudio}

La evaluación de la eficacia de AQ y SP en Nariño se realizó a través de un ensayo clínico aleatorizado no enmascarado, basado en el protocolo estandarizado de la Organización Panamericana de la Salud (OPS) para la "Evaluación de la eficacia terapéutica de los medicamentos para el tratamiento del paludismo por Plasmodium falciparum sin complicaciones en las Américas" (15). La OPS recomienda realizar estos estudios con pacientes representativos de la población afectada por malaria, por lo cual se incluyeron niños mayores de un año. El protocolo del estudio fue evaluado y aprobado por el Comité Institucional de Ética de Investigación en Humanos del CIDEIM antes de su aplicación y se realizó de acuerdo con los principios éticos estipulados en la Declaración de Helsinki (16) y las normas nacionales para la investigación en salud (17).

\section{Sitios y duración del estudio}

El estudio se realizó entre marzo y octubre de 1999 en la población de El Charco y entre mayo de 1999 y abril de 2002 en la población de Tumaco, en el departamento de Nariño al sur de la Costa Pacífica de Colombia. Durante los últimos 4 años, estas dos poblaciones han informado un alto número de casos de malaria con promedios anuales de 1.889 casos en El Charco $(87,2 \%$ por P. falciparum) y 1.033 casos en Tumaco $(81 \%$ por $P$. falciparum).

\section{Inclusión de pacientes}

El reclutamiento de pacientes se realizó en los servicios de diagnóstico de malaria del Hospital San Andrés de Tumaco y del Programa de Control de Enfermedades Transmitidas por Vectores de ambas poblaciones de estudio. Los pacientes con resultado de gota gruesa positivo para infección por $P$. falciparum fueron evaluados para determinar si cumplían con los siguientes criterios de inclusión: edad entre 1 y 65 años, infección pura por $P$. falciparum, parasitemia entre 500 y 50.000 formas asexuales $/ \mathrm{ml}$, disposición para regresar a los controles durante el seguimiento y aceptación de participar en el estudio con la firma del consentimiento informado. No fueron incluidos en el estudio los pacientes con signos o síntomas de malaria grave (18), presencia de enfermedad crónica, embarazo o antecedente de alergia a los antimaláricos. El antecedente de haber tomado tratamiento antimalárico para el episodio actual de malaria no fue considerado como criterio de exclusión.

\section{Tratamientos evaluados}

Los pacientes incluidos fueron asignados al azar por medio de códigos de aleatorización por bloques a uno de los dos tratamientos de estudio: AQ, 25 $\mathrm{mg} / \mathrm{kg}$ divididos en tres días ( $10 \mathrm{mg} / \mathrm{kg}$ los primeros dos días y $5 \mathrm{mg} / \mathrm{kg}$ el tercer día) y SP, $25 \mathrm{mg} / \mathrm{kg}$ de sulfadoxina $1,25 \mathrm{mg} / \mathrm{kg}$ de pirimetamina en dosis única el primer día. Ambos medicamentos provinieron de lotes garantizados por el Ministerio de Salud y fueron iguales a los de uso convencional en el país para el tratamiento de malaria. La administración se supervisó, con repetición de dosis en caso de vómito en los 30 minutos siguientes a la toma. Los pacientes positivos para gametocitos se trataron con primaquina $0,75 \mathrm{mg} / \mathrm{kg}$ en dosis única el último día de seguimiento. Los pacientes que presentaron falla terapéutica a $A Q$ recibieron $S P$ como tratamiento de rescate y los pacientes que presentaron falla terapéutica a $S P$ recibieron $A Q$ como tratamiento de rescate a las dosis descritas anteriormente. Se realizó seguimiento los días 4 
y 7 posteriores al inicio del tratamiento de rescate sin considerar a estos pacientes como nuevos sujetos de estudio.

\section{Seguimiento}

El día de inclusión y de inicio de tratamiento fue considerado como día 0 . Los pacientes fueron citados a controles después de iniciado el tratamiento los días 1, 2, 3, 7 y 14 en El Charco y los días 1, 2, 3, 7, 14, 21 y 28 en Tumaco. El menor tiempo de seguimiento de los pacientes en El Charco se debió a dificultades de tipo logístico y de disponibilidad del personal del estudio en el área endémica. En cada control se realizó evaluación parasitológica por gota gruesa y evaluación clínica para la determinación de desaparición de síntomas o detección de signos y síntomas de complicación. A todos los pacientes se les suministraron toldillos y se recomendó su uso adecuado durante el seguimiento, con el fin de evitar que fueran reinfectados. El uso del toldillo también buscaba evitar que los participantes se convirtieran en reservorio o fuente de nuevas infecciones, ya que no recibieron tratamiento gametocitocida con primaquina sino hasta el final del seguimiento. La respuesta terapéutica fue clasificada como: 1) falla temprana, si el paciente presentaba signos de peligro o malaria grave en los días 1, 2 o 3 con parasitemia; parasitemia del día 2 mayor que la del día 0 o parasitemia del día 3 mayor al $25 \%$ de la parasitemia del día $0 ; 2$ ) falla tardía, si el paciente presentaba signos de peligro o malaria grave después del día 3 con parasitemia; regresaba en día no programado debido a deterioro clínico con parasitemia o presentaba parasitemia en alguno de los días de seguimiento después del día 3 , y 3 ) respuesta adecuada, si el paciente no cumplía con ninguno de los criterios de falla temprana o tardía y la parasitemia desaparecía después del día 3 hasta el día 14 (en El Charco) o 28 (en Tumaco) de seguimiento.

\section{Evaluación parasitológica y control de calidad}

En el día de ingreso y en los días de seguimiento, excepto el día 1, se tomaron dos gotas gruesas que posteriormente fueron teñidas con el método estándar de uso nacional Romanowsky modificado (19) para su lectura en microscopio de luz a
1.000X. La densidad parasitaria en número de formas asexuales $/ \mathrm{ml}$ de sangre se calculó multiplicando el número de formas asexuales de parásitos vistas en 200 leucocitos por 8.000 (número de leucocitos $/ \mathrm{ml}$ de sangre) y dividiendo por 200. Se consideraron como negativas las gotas gruesas en las cuales no se observaron formas asexuales en 200 campos microscópicos revisados. Se realizaron extendidos cada vez con el fin de diferenciar especie en los casos en que se presentó duda. El total de las láminas se leyó una segunda vez por otro microscopista experto, quien desconocía la identidad de las láminas y el resultado inicial de su lectura. Las láminas con resultado discordante (entre positivo y negativo y en recuento parasitario) fueron leídas por un tercer lector y se consideró como verdadero el resultado de uno de los dos primeros lectores más cercano al del tercer lector.

\section{Genotipificación de los casos de falla}

Debido a que el seguimiento de los pacientes en el estudio se realizó por un periodo de tiempo durante el cual podían estar expuestos a nuevas picaduras de mosquitos $\mathrm{y}$, por consiguiente, a reinfecciones, se proveyó a cada paciente de un toldillo con el fin de evitar de que se presentaran fallas terapéuticas por reinfecciones y no por verdaderas recrudescencias. Sin embargo, considerando que la eficacia del uso de toldillo no es del $100 \%$ debido, entre otras cosas, a su uso inadecuado (no hubo verificación del uso adecuado de toldillos), se realizó genotipificación de $P$. falciparum en todos los casos de falla terapéutica con el fin de diferenciar las verdaderas recrudescencias de las posibles reinfecciones. La genotipificación de $P$. falciparum consiste en la amplificación por la técnica de reacción en cadena de la polimerasa (PCR) de los genes $m s p 1, m s p 2$ y glurp, los cuales son altamente polimórficos, lo que permite detectar diferencias entre poblaciones de parásitos. Con esta técnica, el análisis del parásito en el día 0 y en el día de falla permite determinar si el parásito en ambos días fue el mismo (verdadera recrudescencia) o si el parásito del día de falla fue diferente al del día 0 (reinfección). Con este fin, se tomaron muestras de sangre en papel filtro Whatman 3M (Maidstone, Inglaterra) de los días 0 y de falla, se dejaron secar y se 
almacenaron individualmente a temperatura ambiente en bolsas plásticas herméticas hasta su análisis. Se realizó extracción de ADN del papel filtro utilizando el método modificado de Saponina/ Chelex-100 (20) y, posteriormente, se realizó PCR multiplex y anidada para la determinación de las tres familias del gen msp1 (MAD20, RO33 y K1), las dos familias del gen msp2 (IC1/3D7 y FC/ CAMP) y el polimorfismo en tamaño del gen glurp de $P$. falciparum (21). Los productos de la amplificación fueron posteriormente visualizados y comparados en gel de agarosa al 2,5\% (Gibco, Grand Island, NY) teñido con bromuro de etidio $0,2 \mathrm{mg} / \mathrm{ml}$ (Sigma, St. Louis, MO).

\section{Análisis estadístico}

La construcción de la base de datos y su análisis se realizó utilizando el programa Epiinfo, versión 6.04. Se obtuvieron las frecuencias de las variables descriptivas por sitio de estudio y grupo de tratamiento y se calculó la proporción de fallas terapéuticas por sitio de estudio, tratamiento y tipo de falla con el cálculo de intervalos de confianza del $95 \%$. Se utilizó la prueba de $\chi^{2}$ para la comparación de proporciones y se construyeron tablas de 2 × 2 para la determinación de posibles asociaciones entre las variables evaluadas y la presencia de fallas. Se consideró como significativo un valor de $\mathrm{p}$ a dos colas de 0,05.

\section{Resultados}

En total, se incluyeron 106 pacientes en el estudio, 50 en El Charco y 56 en Tumaco. En El Charco, 22 pacientes recibieron tratamiento con $A Q$ y 28 con SP, mientras que en Tumaco 26 pacientes recibieron tratamiento con $A Q$ y 30 con SP. Durante el seguimiento se retiraron 2/50 (4\%) pacientes en El Charco, uno por presentar infección concomitante por $P$. vivax y otro por no poder ser localizado, y 6/56 (10,7\%) pacientes en Tumaco, 5 por no poder ser localizados y uno por retiro voluntario. Los pacientes que tuvieron seguimiento completo y que, por consiguiente, se consideraron en el análisis de las fallas terapéuticas fueron: 22 pacientes con tratamiento con $A Q$ y 26 pacientes con tratamiento con SP en El Charco y 24 pacientes con tratamiento con $A Q$ y 26 pacientes con tratamiento con SP en Tumaco.
Las características demográficas de la población de estudio se presentan en detalle en el cuadro 1. En general, el promedio de edad de los participantes de El Charco fue menor al de los participantes de Tumaco, hubo mayor participación de mujeres en El Charco y la ocupación más frecuente en El Charco fue "estudiante" mientras que en Tumaco fue "agricultor". Hubo diferencias estadísticamente significativas entre EI Charco y Tumaco respecto a los antecedentes de malaria previa y tratamiento previo: en EI Charco fue más frecuente encontrar pacientes que habían tenido malaria previa en el último año $(p<0,001)$ y en Tumaco fueron más frecuentes los casos de pacientes que ya habían tomado $C Q$ para el tratamiento de su episodio de malaria de ese momento $(p=0,03)$.

Por otro lado, no se encontró diferencia en el nivel de parasitemia de ingreso entre las dos poblaciones (cuadro 1). La parasitemia desapareció el día 3 en el $98 \%$ de los pacientes de El Charco y en el $95,8 \%$ de los pacientes de Tumaco. Los síntomas de ingreso más frecuentes en ambas poblaciones fueron fiebre (100\%), cefalea $(96,2 \%)$ y escalofríos (85,8\%). La mayoría del total de pacientes incluidos $(97,9 \%)$ estuvieron afebriles en el día 3 de seguimiento sin diferencias según grupo de tratamiento.

En El Charco no se encontró ninguna falla a ninguno de los dos medicamentos de estudio. En Tumaco, por el contrario, se encontró alto nivel de fallas a AQ (50\% [IC95\%: 30,6 a 69,4]) y menor nivel de fallas a SP (15\% [IC95\%: 5,1 a 33,1]). La mayoría de fallas a SP fueron tempranas (3/4 [75\%]) mientras que la mayoría de fallas a $A Q$ fueron tardías (8/12 [66,7\%]) (cuadro 2). Del total de fallas tardías para ambos medicamentos, 6/9 $(66,7 \%)$ se presentaron después del día 14 de seguimiento. Todas las fallas fueron debidas a verdadera recrudescencia y no a reinfección de acuerdo con los resultados de genotipificación obtenidos (figura 1). No se encontró relación estadísticamente significativa entre la edad $(p=0,6)$, la parasitemia de ingreso $(p=1)$, el antecedente de malaria previa $(p=0,15)$ o el antecedente de tratamiento previo $(p=0,14)$, con la presencia de fallas. 
Cuadro 1. Características demográficas de los participantes en el estudio de la eficacia de amodiaquina (AQ) y sulfadoxina/ pirimetamina (SP) en el tratamiento de malaria no complicada por $P$. falciparum en Nariño, Colombia.

\begin{tabular}{|c|c|c|c|c|c|}
\hline \multirow{2}{*}{\multicolumn{2}{|c|}{ Característica }} & \multicolumn{3}{|c|}{ El Charco $(n=50)$} & \multirow{2}{*}{$\begin{array}{c}\text { Tumaco }(n=56) \\
\text { SP } \\
(n=30)\end{array}$} \\
\hline & & $\begin{array}{c}A Q \\
(n=22)\end{array}$ & $\underset{(n=28)}{S P}$ & $\begin{array}{c}A Q \\
(n=26)\end{array}$ & \\
\hline \multicolumn{2}{|c|}{$\begin{array}{l}\text { Promedio de edad (años) } \\
\text { (rango) }\end{array}$} & $18,4(6-44)$ & $18,9(5-55)$ & $28,6(10-58)$ & $26,4(7-64)$ \\
\hline \multirow[t]{2}{*}{ Sexo (\%): } & femenino & $12(54,5)$ & $17(60,7)$ & $10(38,5)$ & $12(40)$ \\
\hline & masculino & $10(45,5)$ & $11(39,3)$ & $16(61,5)$ & $18(60)$ \\
\hline \multirow[t]{5}{*}{ Ocupación (\%): } & menor de edad & $1(4,6)$ & $5(17,9)$ & 0 & $2(6,7)$ \\
\hline & estudiante & $14(63,6)$ & $12(42,9)$ & $4(15,4)$ & $6(20)$ \\
\hline & hogar & $1(4,6)$ & $7(25)$ & $7(26,9)$ & $5(16,7)$ \\
\hline & agricultor & 0 & $2(7,1)$ & $10(38,5)$ & $7(23,3)$ \\
\hline & otras $^{1}$ & $6(27,3)$ & $2(7,1)$ & $5(19,2)$ & $10(33,3)$ \\
\hline \multicolumn{2}{|c|}{$\begin{array}{l}\text { Tratamiento previo con } \\
\text { cloroquina (\%) }\end{array}$} & $2(9,1)$ & $2(7,1)$ & $8(30,8)$ & $5(16,7)$ \\
\hline \multicolumn{2}{|c|}{$\begin{array}{l}\text { Episodio de malaria en } \\
\text { el último año }(\%)\end{array}$} & $18(81,8)$ & $23(82,1)$ & $7(26,9)$ & $7(23,3)$ \\
\hline \multicolumn{2}{|c|}{$\begin{array}{l}\text { Promedio geométrico de la } \\
\text { parasitemia al ingreso (rango) }\end{array}$} & $10.304(1.702-34.400)$ & $5.970(1.100-24.360)$ & $5.164(600-38.400)$ & $2.079(60-25.200)$ \\
\hline
\end{tabular}

${ }^{1}$ Otras: desempleados y otras ocupaciones menos frecuentes tales como comerciantes y profesionales.

Cuadro 2. Fallas terapéuticas encontradas a amodiaquina y sulfadoxina/pirimetamina en el tratamiento de malaria no complicada por $P$. falciparum en Tumaco, Colombia.

\begin{tabular}{|c|c|c|}
\hline $\begin{array}{l}\text { Respuesta al } \\
\text { tratamiento }\end{array}$ & $\begin{array}{l}\text { Amodiaquina } \\
\qquad(n=24)\end{array}$ & $\begin{array}{l}\text { Sulfadoxina/ } \\
\text { pirimetamina } \\
\qquad(n=26)\end{array}$ \\
\hline alla tem & $\begin{array}{c}4(16,7 \%) \\
\text { [IC95\%: } 5,5 \text { a 35,5] }\end{array}$ & $\begin{array}{c}3(11,5 \%) \\
\text { [IC95\%: } 3,0 \text { a } 28,3]\end{array}$ \\
\hline Falla tardía & $\begin{array}{c}8(33,3 \%) \\
\text { [IC95\%: } 16,8 \text { a } 53,6]\end{array}$ & $\begin{array}{c}1(3,8 \%) \\
{[\text { IC95\%: 0,2 a 17,6] }}\end{array}$ \\
\hline Total fallas & $\begin{array}{c}12(50 \%) \\
{[\text { IC95\%: 30,6 a 69,4] }}\end{array}$ & $\begin{array}{c}4(15 \%) \\
{[\text { IC95\%: } 5,1 \text { a 33,1] }}\end{array}$ \\
\hline
\end{tabular}

\section{Discusión}

LaAQ es un medicamento de reciente introducción formal al esquema de tratamiento de malaria en Colombia por lo cual los altos niveles de falla terapéutica encontrados en Tumaco (50\%) fueron inesperados. La presencia de cepas de $P$. falciparum resistentes a $A Q$ en Tumaco podría deberse a presión de selección por uso previo inadecuado de $A Q$ en la región o a resistencia cruzada con $C Q$ por su analogía estructural. A pesar de la disponibilidad previa de $A Q$ en el país, en la actualidad su distribución es restringida y no se encuentra disponible comercialmente en el país por lo cual las poblaciones de las zonas endémicas tienden a usar más indiscriminadamente la CQ. Este comportamiento hace más posible la hipótesis de la existencia de resistencia cruzada entre ambos medicamentos. El nivel de fallas terapéuticas a $A Q$ en Tumaco estuvo por encima del $25 \%$, porcentaje por encima del cual se considera que el medicamento debe ser retirado y reemplazado por otro de eficacia demostrada (1).

Los estudios realizados en África han demostrado mayor eficacia de $A Q$ a dosis de 30 a $35 \mathrm{mg} / \mathrm{kg}$ sin ser más tóxica que CQ o SP (12). La dosis de $A Q$ recomendada en la actualidad en el país es de $25 \mathrm{mg} / \mathrm{kg}$, la cual puede ser insuficiente en el tratamiento de malaria por $P$. falciparum resistente a CQ. El uso de $A Q$ a la dosis actual para el tratamiento de malaria en Colombia debe ser reevaluado considerando el uso de dosis más altas como una alternativa para mejorar su eficacia y prolongar su tiempo de vida útil en el país.

Los bajos niveles de fallas terapéuticas a SP encontrados en Tumaco (por debajo del 25\%) permiten continuar su uso en el tratamiento de malaria por $P$. falciparum en Colombia. Sin 


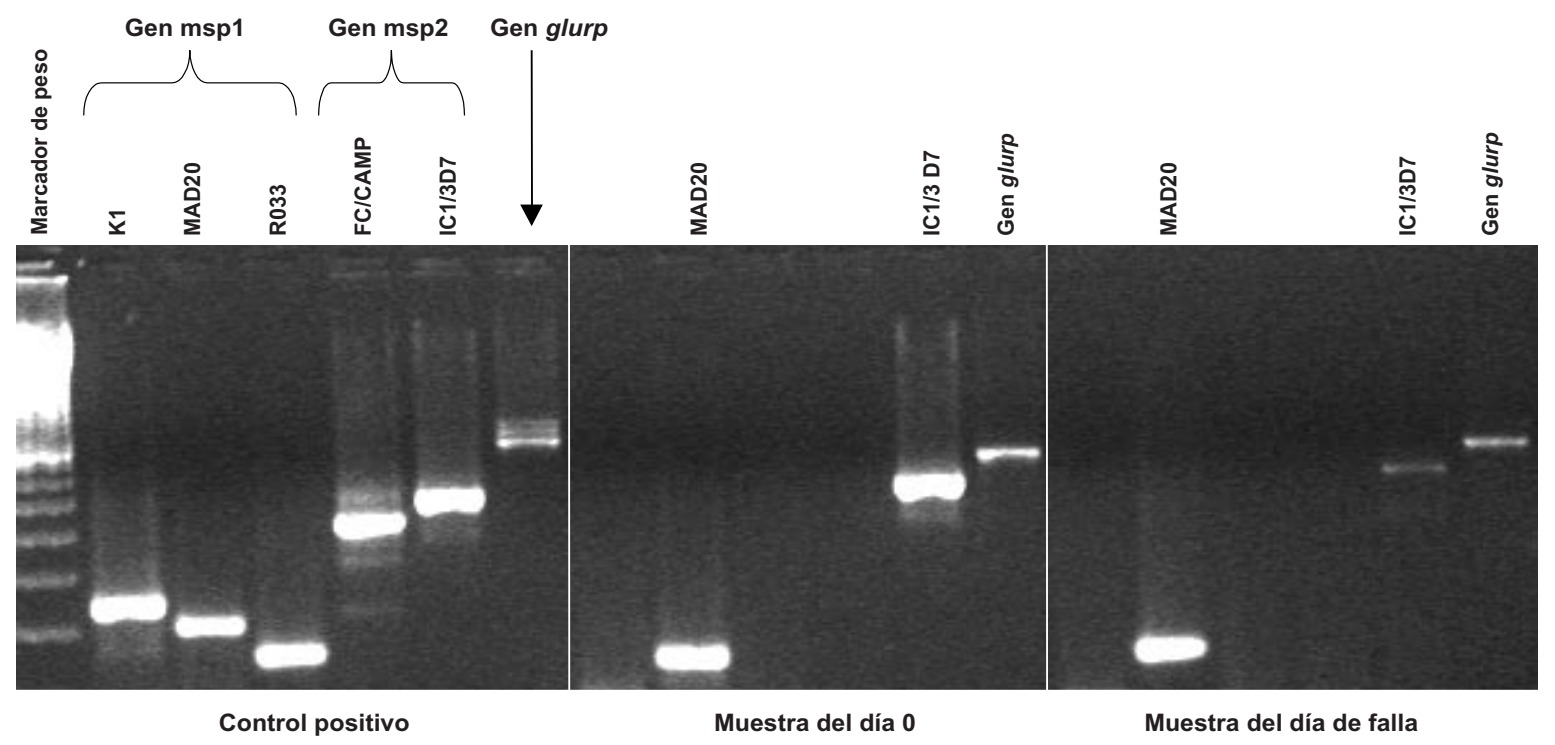

Figura 1. Genotipificación de $P$. falciparum en la diferenciación de verdadera recrudescencia de reinfección: el control positivo muestra las bandas de amplificación de las tres familias del gen msp1 (K1, MAD20 y RO33), de las dos familias del gen $m s p 2$ (FC/CAMP e IC1/3D7) y del gen glurp. Las bandas que se observan en la muestra del día 0 evidencian la presencia de la familia MAD20 de $m s p 1$, la familia IC1/3D7 de $m s p 2$ y una banda de amplificación en glurp. La muestra del día de falla evidencia la presencia de las mismas familias con bandas de igual peso molecular a las del día 0 , lo cual permite concluir que el parásito del día de falla es igual al del día 0.

embargo, el aumento en el nivel de fallas con respecto a lo encontrado en años previos y la predominancia de fallas tempranas $(75 \%)$ indican que el uso continuo de este medicamento y su vida media larga han contribuido en el aumento de cepas resistentes por presión de selección. Es necesario continuar vigilando la eficacia de SP de manera sistemática y promover decididamente su uso combinado con otros antimaláricos eficaces con el fin de preservar su vida útil en el país.

Contrario a lo encontrado en Tumaco, no se detectaron fallas a los medicamentos en estudio en El Charco. Un factor determinante de esta diferencia pudo ser el tiempo de seguimiento de los pacientes en El Charco, lo que impidió detectar fallas después del día 14, más si se tiene en cuenta la alta proporción de fallas tardías que fueron detectadas después del día 14 en Tumaco $(66,7 \%)$.

Otros factores de tipo epidemiológico podrían también estar determinando las diferencias en el nivel de fallas entre ambas poblaciones: el predominio de casos en estudiantes en El Charco podría ser un indicador de transmisión urbana en esta población, mientras que el predominio de casos en agricultores en Tumaco indicaría un tipo de transmisión rural. El flujo de población en Tumaco, por cuestiones económicas y comerciales, podría estar favoreciendo la creación de focos de resistencia a partir de infecciones provenientes de otras áreas (22). Por otro lado, el antecedente de malaria previa en más del $80 \%$ de los casos en El Charco sugiere que las altas tasas de transmisión en esta región confieren inmunidad parcial, que favorece la eliminación de cepas resistentes en nuevas infecciones, mientras que la alta frecuencia de casos con antecedente de tratamiento previo con $C Q$ en Tumaco podría estar demostrando el uso inadecuado de este medicamento, lo cual favorece la resistencia cruzada con $A Q$.

La genotipificación de $P$. falciparum para la determinación de verdaderas recrudescencias en áreas en donde existe el riesgo de reinfección en pacientes en seguimiento ha sido una herramienta 
de uso amplio en estudios de eficacia de antimaláricos (23-25). El uso simultáneo de tres genes diferentes y no asociados, como son msp1, msp2 y glurp, robustece la prueba porque permite diferenciar con mayor precisión diferentes clones de parásitos por infección, principalmente en áreas de alta transmisión en donde las poblaciones de parásitos son bastante heterogéneas (23). Los bajos niveles de transmisión en Colombia (2) y los estudios previos de genotipificación de cepas colombianas con otros genes (26) sugieren que las poblaciones parasitarias de las zonas endémicas del país son muy homogéneas. Los análisis de genotipificación del presente estudio dieron como resultado que todas las fallas encontradas se debieron a verdaderas recrudescencias. Sin embargo, es posible que algunas de las fallas encontradas, principalmente las tardías, puedan haberse presentado por reinfecciones con poblaciones homogéneas de parásitos que no fueron discriminadas con esta metodología.

El uso de terapias combinadas ha sido recomendado a nivel mundial como estrategia para retardar el surgimiento y dispersión de la resistencia (27). En Colombia, desde hace varios años se recomendó el uso de combinaciones, inicialmente de CQ con SP y posteriormente de $A Q$ con SP $(3,28)$. Aparentemente, la eficacia de SP ha estado protegida por esta estrategia, sin embargo, el uso popular inadecuado y como monoterapia de la CQ favoreció el surgimiento de altos niveles de fallas a este medicamento y su retiro de las normas oficiales de tratamiento de malaria del Ministerio de Salud.

La utilidad del uso de combinaciones depende tanto de la disponibilidad de los medicamentos en el momento de usarlos como de la eficacia demostrada de ambos, ya que el efecto de uno puede enmascarar la ineficacia de otro. El uso de $S P$ en combinación con $A Q$ en áreas en las cuales los niveles de falla a $A Q$ son altos tendrá el mismo efecto que usar SP sola, desprotegiéndola y favoreciendo el aumento de fallas a SP. Los altos niveles de falla terapéutica a $A Q$ encontrados sugieren que su uso a las dosis actuales en combinación con SP tendrá un tiempo de vida útil más corto que el esperado. Estrategias tales como la evaluación de la sensibilidad in vitro de cepas locales de $P$. falciparum y la determinación de la eficacia in vivo de nuevos antimaláricos, nuevas dosis o nuevas combinaciones de ellos antes de su introducción oficial al país deben ser consideradas en el momento de tomar decisiones respecto a las normas de tratamiento de malaria en Colombia.

\section{Agradecimientos}

Al personal del Programa de Control de Enfermedades Transmitidas por Vectores del departamento de Nariño y al personal del Hospital San Andrés de Tumaco, especialmente a su director, Jesús Quiñones, y a la bacterióloga, Patricia Orobio, por su colaboración en la conducción del estudio. A Ricardo Palacios, Beatriz Ferro, Luba Pardo y Wilson Cortés, personal de CIDEIM que colaboró en diferentes fases de la ejecución del estudio y a José R. Tovar por su colaboración en el análisis estadístico de la información. Este estudio se realizó con el apoyo financiero de COLCIENCIAS (Proyecto 2229-04626-98) y del Programa Especial para Investigación y Entrenamiento en Enfermedades Tropicales de UNDP/World Bank/WHO (ID: 981015).

\section{Referencias}

1. World Health Organization. The use of antimalarial drugs. Report of a WHO informal consultation. 2001. WHO/CDS/RBM/2001.33. http://www.who.int/en/

2. Pan American Health Organization. Report of the status of malaria programs in the Americas (Based on 2000 data). 2001. CD43/INF/1. http://www.paho.org/English/GOV/CD/cd43-inf1-e.pdf

3. Ministerio de Salud de Colombia. Guía de atención médica para el diagnóstico y tratamiento de la malaria. 2000. Resolución $N^{\circ} 0412$, cap. 37. http:// www.minsalud.gov.co/NewSite/MseContact/

4. Organización Panamericana de la Salud. Situación de Salud en Colombia. 2002. http://www.col.opsoms.org/default.asp

5. Osorio LE, Giraldo LE, Grajales LF, Arriaga AL, Andrade AL, Ruebush TK, et al. Assessment of therapeutic response of Plasmodium falciparum to chloroquine and sulfadoxine-pyrimethamine in an area of low malaria transmission in Colombia. Am J Trop Med Hyg 1999;61:968-72.

6. Blair-Trujillo S, Lacharme-Lora L, CarmonaFonseca J. Resistance of Plasmodium falciparum to 
antimalarial drugs in Zaragoza (Antioquia, Colombia), 1998. Mem Inst Oswaldo Cruz 2002;97:401-6.

7. Castillo CM, Osorio LE, Palma GI. Assessment of therapeutic response of Plasmodium vivax and Plasmodium falciparum to chloroquine in a malaria transmission free area in Colombia. Mem Inst Oswaldo Cruz 2002;97: 559-62.

8. Blair S, Lacharme LL, Carmona J, Tobón A. Resistencia de Plasmodium falciparum a tres fármacos antimaláricos en Turbo (Antioquia, Colombia), 1998. Pan Am J Public Health 2001;9:23-9.

9. World Health Organization. Antimalarial drug combination therapy. Report of a WHO technical consultation. 2001. WHO/CDS/RBM/2001.35. http://www.who.int/en/

10. Brasseur $\mathbf{P}$, Guiguemde $\mathbf{R}$, Diallo $\mathbf{S}$, Guiyedi $\mathbf{V}$, Kombila M, Ringwald $P$, et al. Amodiaquine remains effective for treating uncomplicated malaria in West and Central Africa. Trans R Soc Trop Med Hyg 1999;93:64550.

11. Espinal CA, Cortes GT, Guerra P, Arias A. Sensitivity of Plasmodium falciparum to antimalarial drugs in Colombia. Am J Trop Med Hyg 1985;34:675-80.

12. Olliaro P, LeBras J, Ringwald P, Mussano P, Garner $\mathbf{P}$, Brasseur P. Systematic review of amodiaquine treatment in uncomplicated malaria. Lancet 1996;348:1196201.

13. Ringwald P, Bickii J, Basco LK. Amodiaquine as the first-line treatment of malaria in Yaoundé, Cameroon: presumptive evidence from activity in vitro and crossresistance patterns. Trans R Soc Trop Med Hyg 1998; 92:212-3.

14. Ministerio de Salud de Colombia. Red Nacional de Vigilancia Centinela de la Resistencia a los Medicamentos Antimaláricos. Presentación Plan de Trabajo. I reunión técnica de coordinación de la Red Amazónica de Vigilancia de la Resistencia a los Antimaláricos (RAVREDA) y de la Iniciativa de Malaria en la Amazonía (AMI). Santa Cruz, Bolivia. Marzo 19 y 20 de 2002. http:/ /www.paho.org/Spanish/HCP/HCT/MAL/ reunion2002.htm

15. Organización Panamericana de la Salud. Evaluación de la eficacia terapéutica de los medicamentos para el tratamiento del paludismo por Plasmodium falciparum sin complicaciones en las Américas, 1998. OPS/HCP/ HCT/113/98. http://www.paho.org/Spanish/HCP/HCT/ doc452.pdf

16. World Medical Association Declaration of Helsinki. Ethical principles for medical research involving human subjects. Last revision. 52 ${ }^{\text {nd }}$ WMA General Assembly, Edinburgh, Scotland. October 2000. http://www.wma.net/ e/policy/17-c_e.html

17. Ministerio de Salud de Colombia. Normas científicas, técnicas y administrativas para la investigación en salud. 1993. Resolución No. 008430. http:// www.minsalud.gov.co/NewSite/MseContact/

18. World Health Organization. Management of uncomplicated malaria and the use of antimalarial drugs for the protection of travelers. 1996. WHO/MAL/96.1075. http://www.who.int/en/

19. López F, Schmunis G. Diagnóstico de malaria. Publicación científica $N^{\circ}$ 512. Washington, D.C.: Organización Panamericana de la Salud; 1988. p.84.

20. Giraldo LE, Acosta MC, Labrada LA, Praba A, Montenegro-James S, Saravia NG, et al. Frequency of the Asn-108 and Thr-108 point mutations in the dihydrofolate reductase gene in Plasmodium falciparum from southwest Colombia. Am J Trop Med Hyg 1998;59: 124-8.

21. Viriyakosol S, Siripoon N, Petcharapirat C, Petcharapirat $\mathrm{P}$, Jarra $\mathrm{W}$, Thaithong $\mathrm{S}$, et al. Genotyping of Plasmodium falciparum isolates by the polymerase chain reaction and potential uses in epidemiological studies. Bull World Health Organ 1995;73:8595.

22. Martens $\mathbf{P}$, Hall L. Malaria on the move: human population movement and malaria transmission. Emerg Infect Dis 2000;6:102-9.

23. Snounou G, Beck HP. The use of PCR-genotyping in the assessment of recrudescence or reinfection after antimalarial treatment. Parasitol Today 1998;14:462-7.

24. Ohrt C, Mirabelli-Primdahl L, Karnasuta C, Chantakulkij S, Kain K. Distinguishing Plasmodium falciparum treatment failures from reinfections by restriction fragment length polymorphism and polymerase chain reaction genotyping. Am J Trop Med Hyg 1997;57: 430-7.

25. Brockman A, Paul RE, Anderson TJ, Hackford I, Phaiphun L, Looareesuwan S, et al. Application of genetic markers to the identification of recrudescent Plasmodium falciparum infections on the northwestern border of Thailand. Am J Trop Med Hyg 1999;60:1421.

26. González IJ, Varela RE, Murillo C, Ferro BE, Salas J, Giraldo LE, et al. Polymorphisms in cg2 and pfcrt genes and in vitro resistance to chloroquine and other antimalarials in Plasmodium falciparum isolates from Colombia. Trans R Soc Trop Med Hyg 2003 (en prensa).

27. White NJ. Delaying antimalarial drug resistance with combination chemotherapy. Parassitologia 1999;41: 301-8.

28. Ministerio de Salud. Guía integral de manejo de las enfermedades transmitidas por vectores. Malaria, dengue y leishmaniasis. Unidad Administrativa Especial de Campañas Directas. 1986. http://www.minsalud.gov.co/ NewSite/MseContact/ 